\title{
SATISFACTION WITH ENVIRONMENT OF REGIONAL HEALTH CARE INSTITUTION: PATIENTS' OPINIONS AND SOCIETAL STEREOTYPES
}

\author{
Arturs Medveckis ${ }^{1}$, Dr. paed.; Tamara Pigozne ${ }^{2}$, Dr. paed., \\ ${ }^{1}$ Liepaja University; ${ }^{2}$ University of Latvia
}

\begin{abstract}
Several factors determine selection of a medical institution: demand, accessibility, quota system, distance from residence etc. However, patients' subjective attitude towards the medical system and especially towards the medical establishment is also important, which is optimal in terms of accessibility. Patients' attitude depends on the direct personal experience which has been obtained in the particular medical establishment. Information found by patients, while communicating with each other in social environment, is important, similarly encountering media environment and communication in social networks. Negative information is sometimes perceived or even stereotypes cultivated without direct experience, which contrast the true situation.

To assess patients' opinion on Regional Hospital's environment, extensive studies were carried out in one of Latvia Regional Hospitals during 2007 and 2016, based on direct information acquisition from patients who received a particular medical service cycle in Admission Department, also when discharging from certain profile departments. During the research confirmation was obtained that patients assess objectively provided service quality, express attitude towards different aspects of physical and social environment. Comparative studies provide patients' opinion in dynamics about satisfaction with Regional Hospital's environment, medical staff's attitude towards patients. To make sure improvements of Regional Hospital's environment and service quality are objectively assessed in social environment, qualitative research was carried in 2018 to find out whether in the region living potential patients' opinions reflect the real situation, and whether negative stereotypes exist. Conclusion made that respondents have overcome negative stereotypes, essential improvements of service quality have been noticed objectively, affecting physical and social environment.
\end{abstract}

Key words: satisfaction, hospital environment, quality of service

JEL code: R58, I31

\section{Introduction}

The state protects people's health (The Constitution of the Republic of Latvia, 1922). Stating and implementing the policy and activities of all communities, a high level of people's health protection has to be provided (The consolidated version of the contract about Foundation of the European Union and European Community, 2007). Improvement of patients' service is one of healthcare responsibilities. Patients/clients' well-being is greatly affected not only by the process and outcomes of the treatment or provided service, but also by the access to the service, culture of communication, as well as the environmental accessibility and quality. Information on how patients assess the work of healthcare institutions: what gives them satisfaction, what claims there are, what expectations there were and the real assessment (of expectations) based on the personal experience, can facilitate development of particular improvement measures.

In recent decades the interest in patients' satisfaction with the quality of healthcare and environment as its essential criterion has increased (Valencia-Arias et al., 2018). Patient's satisfaction as the individual's positive assessment about different healthcare dimensions (LinderPelz, 1982) or assessment of the particular service received includes cognition and emotional reactions (Fitzpatrick, 1997). In its turn, the quality of service is a measurement of how satisfactorily the delivered service can meet the standard or justify the client's expectations (Azmi et al., 2018). The quality of service has been analysed in five dimensions.

- Tangibility is the physical evidence of the service, e.g. appearance of tools, gadgets and physical facilities, applied to provide the service.

\footnotetext{
1 arturs.medveckis@liepu.Iv

2 tamara.pigozne@lu.lv
} 
- Reliability is the ability to perform the promised service in an accurate, precise and neat way.

- Responsiveness is the employees' readiness and willingness to assist customers, providing them with services in good time.

- Assurance is the employees' knowledge and ability to have trust and confidence in customers.

- Empathy is caring, individualized and customized attention to the customer (Parasuraman et al., 1985).

The framework of the integrative quality is based on the model of the holistic quality system, which consists of 3 domains related to quality:

- Paying attention to the manufactured attributes/products, their quality and design during the production process;

- Focusing on how the manufacturer produces and provides services during the experience process, which is affected by patients' knowledge, motivation and emotions;

- Emphasizing the quality assessment during the assessment process according to clients' hopes and expectations (Golder et al., 2012).

The criteria of the healthcare quality identified in the critical review are accessibility of services, communication with the staff, care workers' knowledge and skills, hospital staff's responsiveness, responsibility, attitude, behaviour, flexibility, dignity, faithfulness, privacy, pain management, information, cleanliness, silence etc. (Ferrand et al., 2016), also modern and efficient technologies (Okpala, 2018), social responsibility (Radu, 2018), and flexibility of service convenience (Sergio, 2015; Ponsignon et al., 2015; Kumar et al., 2017).

Service convenience in the healthcare context is characterized by the ability to react flexibly to the patient's desires in order to provide a convenient and efficient service. There is a correlation between patients' satisfaction and service convenience flexibility, concentrating on the delivery place and time, new, unique and innovative value-added services, different registration modes in the healthcare delivery system, etc. (Kumar et al., 2017).

The outcomes of the research confirm that the emotional intellect is one of the patients' determinant factors of satisfaction - a positive and statistically significant relationship between "the satisfaction scores and emphatic concern, utilization of emotions, and emotional awareness subheadings of the patients" (Celik, 2017, 1363), doctors' empathy (Wang et al., 2018) and nurses' leadership styles (Sfantou et al., 2017) has been identified.

In 2007 and 2016, a study was carried out in one of regional hospitals of Latvia in order to find out patients' opinions on the services, course of treatment, medical staff's attitude provided in the hospital in order, applying the obtained outcomes of the research analysis, assess claims, identify possible insufficiencies in patients' service and improve the system of measures in the hospital work development, in order to analyse patients' satisfaction with the service quality provided by the hospital in dynamics. One of the reasons for a repeated study was the reconstruction carried out in the hospital (from February 2011 till September 2014). After the reconstruction not only the outer appearance has changed, but also the infrastructure has been improved, better energy efficiency achieved, indoor aesthetic environment has changed and functionality of the rooms available for patients enhanced. Standard departments with a modern planning have been built. Also, new equipment for diagnostics and treatment has been obtained. When implementing the reconstruction of the hospital, lots of attention has been paid to both the hospital staff and clients' convenience. The focus of the research has been directed towards the assessment of Environmental Improvements on patients/ clients' well-being. 
The outcomes of the research cannot be related to the work of healthcare institutions in the whole country, they only reflect the patients/clients' assessment in the particular regional hospital. Findings of the research cycle have been used for service culture enhancement and patients/clients' satisfaction increase. Such an approach can also be applicable for the assessment of own work and environmental culture organization in other healthcare institutions.

Goal of the research: analyse in dynamics patients/clients' opinions and society's stereotypes about the satisfaction with the regional hospital's environment.

In 2007 and 2016 research methodology was developed studying and adapting to local needs the applied methods of the World Health Organization's (WHO) hospital performance assessment project "Performance Assessment Tool for Quality Improvement in Hospitals (PATH)" and the selfassessment model used in US hospitals „Consumer Assessment of Healthcare Providers and Systems (CHAPS)".

The empirical methods applied in the research:

- Data acquisition methods (survey and focus group discussions);

- Data processing methods Kolmogorov-Smirnov's Test for the empirical division determination, Mann-Whitney $\mathrm{U}$ Test and Kruskal-Walis $\mathrm{H}$ Test for the difference determination between the criteria and indices depending on the respondents' profile, Kendall's Tau-b Test for the coherence determination and Chi-Square for the comparison of 2007 and 2016 outcomes for the quantitative data processing of the programme in the SPSS environment.

The research has been implemented in 3 stages:

- Stage 1 from June 1 till July 1, 2007;

- Stage 2 from July 5 till September 14, 2016;

- Stage 3 from September 10 till October 10, 2018.

In the survey, the questions were included which reflect information on the length of treatment, number of patients in a ward, staff's culture of communication, accessibility of the physical environment, informative environment etc., which enabled to obtain a direct respondents' subjective point of view about the quality of service, satisfaction with services, doctors' attitude, satisfaction with the infrastructure and environment applicable an available for the hospital patients etc.

The respondents' selection was determined according to the nesting principle in order all departments of the medical institution would be represented, determining the number of respondents in the selection proportionally to the number of patients in the full previous year, within the margin of a $5 \%$ tolerable statistical error.

In Stage 1 of the research, 444 respondents (patients) took place, but in Stage 2 of the research 483 respondents (patients) participated, who during the survey were discharged from the hospital:

- 137 men and 346 women;

- 303 towns where the regional hospital, inhabitants are located; 89 regional inhabitants and 91 inhabitants from other regions;

- 18 aged 14-18, 143 aged 19-39, 180 aged 40-65, 152 older than 66.

In Stage 3 of the research, 9 respondents of each focus group took part in the interviews, modelling the represented respondents' profile according to the age, gender and division of residence, but they are the people who have not used the hospital services for at least 2 years.

In the daily informative environment, we sometimes encounter an opinion expressed by individual people on topics topical to the society, including accessibility of medical institutions, quality of 
treatment, service and medical staff's attitude to patients. The most active commentators of the informative reports or articles are sometimes the sceptically-orientated ones.

With the help of the focus group interview there has been set a goal in the research to find an answer to the question whether in the social environment stereotypes are generated about the quality of the institution's medical and physical environment which has not been rooted in the personal experience?

In data of 2007 the reflected gender proportion confirms that there are women almost twice as more $(65.5 \%)$, whereas the data of 2016 mark slight changes, where the proportion of women among the patients increases up to $71.4 \%$.

Two essential aspects characterize the accessibility of healthcare: the financial one - people's opportunities to use healthcare services depending on their financial means, and the accessibility possibilities to get to the doctor, distance to the healthcare institution, as well as the opportunity to receive medical services (doctor's availability and working hours). The regional hospital offers medical services to anybody, whoever needs them. However, they are basically used by the city inhabitants of the regional hospital's location and people of its nearest rural territory and towns within $100 \mathrm{~km}$ radius. Similarly to the survey in 2007, the people living in the regional city dominate in the patients' flow - $62.7 \%$ (in 2007- 73.4\%). The number of patients who do not live in the particular region has increased: if in 2007 there were $4.3 \%$ such respondents, then in 2016 there were already $18.8 \%$ such respondents. This leads to the conclusion that the services of Regional Hospital are chosen not only by the inhabitants who live nearby, but also by patients who have chosen their treatment purposefully just in the particular hospital and not in the medical institution closest to their place of residence.

Most patients (72 \%) still received their treatment in Regional Hospital for the first time and their assessment of the hospital's service culture was not influenced by the previous experience. In 2007 there were $66.9 \%$ such patients. The patients, who have been to the hospital for the second time or several times, most probably, express their subjective assessment comparing it with their previous experience, which in most cases, assessing in dynamics, is with a positive tendency.

In 2007 on average patients were treated in the hospital for 21 days, but in 2016 the length of treatment decreased by 6 days. The decrease of the days spent in hospital confirms the successfully introduced approach in the treatment process, patients more often go home, but they only go to hospital when there are planned treatments, check-ups, they stay at the day care unit.

According to the data of Survey in 2016, $23 \%$ of respondents received their treatment in a singleroom ward, in comparison with the data of Survey in 2007 , the number has decreased by $3.1 \%$. The number of patients who received their treatment in a double-room ward has slightly changed: the results of Survey in 2016 are similar to the results of Survey in 2007 (in $201635.4 \%$ respondents, in $200735.6 \%$ respondents). Comparing the research outcomes of Stage 1 and 2, the number of patients placed in triple-room wards has increased essentially ( $p \leq 0.05)$. If in 2007 only $16 \%$ of the surveyed patients were treated in a triple-room wards, then in $201638.9 \%$ of patients were treated in a triple-room ward. The number of patients has decreased who were treated in a quadruple and more room wards. In 2007 there were $13.7 \%$ such respondents, but in 2016 - only $2.1 \%$ respondents. Also, the number of patients who were more than four in a ward has decreased: in Survey 2016 the number has decreased from $8.6 \%$ (in 2007) to $0.4 \%$ (in 2016). 


\section{Research results and discussion}

Admission Department is sometimes the first place the patient encounters when gets to hospital. It is a place where the first aid is provided and the patient's health problems are assessed in order to refer patients further for an appropriate treatment. The patient makes a direct first impression just about Admission Department.

In order to find out satisfaction with the quality of service in Admission Department, the patient was asked to give their subjective assessment, as well as assess the timely help provision, show to what extent they are satisfied with the services of Admission Department. The outcomes of the survey prove that $74.8 \%$ of respondents are satisfied with Admission Department services, but $19.4 \%$ of respondents are partly satisfied.

There is a statistically significant correlation ( $p \leq 0.05 ; r=0.83$ ) between the quality assessment of Admission Department services and the time spent in Admission Department - most of the respondents who have spent in Admission Department less than $30 \mathrm{~min}$ are satisfied with the quality of services, whereas the most dissatisfied ones are the respondents who have spent in Admission Department more than two hours. Respondents' satisfaction with the quality of Admission Department's services is inversely proportional to the time spent in Admission Department - it increases when the time spent in Admission Department decreases, and it decreases when the time spent in Admission Department increases. Quite logical is the tendency: the more time is spent in Admission Department, the lower assessment is received for the quality of the obtained service $(p \leq 0.05)$.

In the comments written by patients the long waiting time has been mentioned as a reason for dissatisfaction. It can be concluded that dissatisfaction is caused not by the quality of service (staff's attitude, quality of the performed treatments, professionalism, quality of help provision etc.), but rather the long time spent in Admission Department.

There is a statistically significant difference $(p \leq 0.05)$ between the time spent in Admission Department in 2007 and 2016, marking a positive dynamics: the number of patients who have spent there up to $30 \mathrm{~min}$ has increased essentially, so decreasing the number of patients who have spent there more than 2 hours.

The results of Mann-Whitney U Test confirm a statistically significant difference $(p \leq 0.05)$ in the quality assessment of Admission Department's services depending on the respondents' gender males are tended to assess it higher (Mean rank=232.89) than females (Mean rank=209.68).

The results of Kruskal-Walis H Test confirm a statistically significant difference $(p \leq 0.05)$ in the quality assessment of Admission Department's services depending on the respondents' age respondents aged 14-18 are tended to assess it higher (Mean rank=235.09) in comparison with the respondents aged 19-39 (Mean rank=200.58).

Most or $88.2 \%$ of the surveyed patients have assessed the quality of services provided by Regional Hospital as positive. The data of Survey 2016 have marked a positive tendency in its assessment - a statistically important difference $(p \leq 0.05)$ has been noticed between the satisfaction with the quality of services provided by Regional Hospital in 2007 and 2016 - the number of the respondents who are completely satisfied with it has increased.

Patients' satisfaction with the provided service quality is related to awareness of the quality of the surrounding environment, as well as assessment of the social environment which depends on the mutual medical staff and patient's communication culture, as well as on the systemic standard congruity or incongruity with patients' expectations, for instance, waiting time to receive services. 
The reasons for dissatisfaction with the quality of the regional hospital's services in 2016 are mentioned such as food (the reason for dissatisfaction 30 times). It is likely that the assessment has not been affected by the quality of the food, which complies with the set diagnosis requirements, but rather their eating habits, family traditions etc., and this is also confirmed with the comments expressed by patients: vegetarian food, fresh fruit and vegetables should also be included in the offer. For some patients the food seemed too salty, for others too small portions. Some patients have expressed their dissatisfaction with the doctors' attitude (mentioned 6 times as the reason for dissatisfaction). On very few occasions the reason for dissatisfaction was the nurses' attitude, quality of the performed tests (mentioned as a reason for dissatisfaction 3 times) and access to the necessary objects in rooms, corridors, treatment rooms, lift, etc. (mentioned as a reason for dissatisfaction twice).

As a recapitulative hospital service assessment could be used the respondents' reply to the question whether they would recommend the hospital to others or whether they themselves would choose it again in case of a necessity.

The outcomes of Survey 2016 prove that $89.5 \%$ of respondents would recommend the services of Regional Hospital to their relatives, friends, acquaintances, but $86.4 \%$ of the respondents would choose the hospital again in case of a necessity.

In 2016, a positive tendency was observed in comparison with 2007 - the number of patients has increased who have positive feelings regarding the service culture and environment in general, and in case of a necessity, they would be ready to recommend this hospital to their relatives, friends and family members, also, if there was such a need, they would be ready to receive treatment for themselves there.

The outcomes of Kruskal-Walis $\mathrm{H}$ Test confirm that there is a statistically significant difference $(p \leq 0.05)$ between the opinion whether Regional Hospital would be chosen as a place for treatment and respondents' place of residence in case of a necessity. A positive answer has been given by respondents living somewhere else (Mean rank=267.90) in comparison with the respondents living in the city where Regional Hospital is located (Mean rank=220.58).

In general, the outcomes of Survey 2016 reflect a very positive assessment of the hospital and staff's activity. Several indices prove that, especially the respondents' positive attitude, expressing an assumption that in case of a necessity they would choose Regional Hospital for treatment purposes and would also recommend others to do it.

In the interviews of the focus groups opinions were found out about the environment of Regional Hospital from the point of view of the people who have not used the hospital services recently, which was important in the research in order to find out who and what way creates the attitude towards the hospital environment.

In the epitome of the interviews it was revealed that the social experience developed historically is sometimes related to the preconceptions about the current situation what it is like in Regional Hospital: I had received treatment in Riga Old Clinic. The atmosphere was depressing. Buildings reminded me of a museum. Cramped for space and overpopulation. I could not imagine that our Regional Hospital would change my impressions about the hospital environment. That is true, one can get lost at first if a room has to be found. (Male, 24, city dweller)

People of rural areas, who have lost their opportunities to receive certain medical services in the nearest medical institution, consider distance as the main problem, paying less attention to the aesthetic or functional environmental factors of the renovated hospital: For me it would be better if 
I could go to my hospital with my health problems, but there are No more such departments and specialists. Now I have to drive $60 \mathrm{~km}$ from my house. Everything is alright if you make an appointment in advance and wait for your turn, but getting there is difficult. (Female, 35, town dweller)

The developed infrastructure has been approved which preserves personal privacy: I really do not feel like putting my problems for everybody on a tray. It used to be very annoying when some time ago I had to sit at a door on which it was written what kind of room it was. Now only the number of the room is written on the door and on my appointment note. (Female, 46, city dweller)

Technologies and informative resources of the $21^{\text {st }}$ century are important achievements. However, possibilities of face-to-face communication are appreciated highly: For an elderly person it is not easy to deal with the room numbers unless nurses help them. It is hard to follow the lights when they are on and when you can go in or when you have already missed your turn. No way I could manage it without my daughter's help. (Female, 79, rural resident)

People, who have encountered services of medical institutions for several decades, can assess objectively the changes of the environment and service culture: I have faced hospital several times during my lifetime. It cannot be compared with the appearance of the newly-built hospital 30 years ago and now. When my first child was born, it was another time. I have had to visit my relatives recently. It is better than in a hotel. (female, 50, city dweller)

The assumption that negative stereotypes have got an essential importance was not approved. Nowadays informative space provides fast circulation of information. However, negative comments are quite often reproduced in news portals by a limited range of people. The assumption, that the people who are not the medical service recipients themselves, are affected by the information and manifested attitude of the closest people's circle has been partly approved. Moreover, stereotypes are generated by the opinions expressed in the mass media, which may not coincide with the immediate information, obtained in the quantitative study straight from the service recipients, that has been obtained straight after the discharge from hospital.

\section{Conclusions, proposals, recommendations}

1) The assessment of Regional Hospital's physical and social environment has been positive in general from the patients' point of view both in research 2007 and 2016 . The positive assessment in dynamics is even more distinct that was affected by the modernization and infrastructure development of Regional Hospital, which provides a better service accessibility for patients and enables the hospital to implement higher quality standards in health care.

2) The proactive action of Regional Hospital's administration has promoted high standard improvement of the social environment and communication culture.

3) The relatively low number of dissatisfied patients has been assessed as a special case. However, it has been a ground for negative comments presence in the informative space. In Stage 1 of the research existence of separate negative impressions was affected by the negative social and personal experience developed historically during several decades- stereotypes, which were associated with the environment of medical institutions in different curative establishments. In Stage 2 of the research it was possible to notice that the presence of negative stereotypes has decreased essentially, which can be explained with the proportional increase of the new patients' generation whose personal experience is not related to the negative past heritage.

4) In group interviews, which were specially directed to find out the existence of stereotypes, it has been discovered that also the people, who have not encountered the regional hospital's 
environment recently, do not represent any stereotypes in general, which could have been met ten or more years ago, but they make objective judgements about the environmental coordination and on separate occasions they refer to the problems which emerge from the patient's individual desires or systemic problems as well, determined by the service availability quota and the waiting time related to it.

5) The stereotypes noticed during the last decade and earlier cultivated myth about the dissatisfactory or partly satisfactory hospital environment were decreased by the objective improvements of the situation, which are appreciated by any service recipient and the approval received from the professional medical staff's associations, Latvian Medical Association available of the public information space.

6) The outcomes of the research cannot be related to the work of healthcare institutions in the whole country, they only reflect the patients/clients' assessment in the particular regional hospital. Findings of the research cycle have been used for service culture enhancement and patients/clients' satisfaction increase. Such an approach can also be applicable for the assessment of own work and environmental culture organization in other healthcare institutions. Systemic and systematic monitoring of patients' satisfaction and the desire to improve the service quality is an essential tool for patients' satisfaction enhancement. Its introduction in all medical service provision institutions would be a highly recommended measure.

\section{Bibliography}

1. Azmi, T., Rahman, H., Mustafi, M.A.A., Islam, R. (2017). Measurement of Patient Satisfaction with SERVQUAL Model of Private Hospitals: SEM Approach. American Journal of Management, 17(3), 64-76.

2. Celik, G.O. (2017). The relationship between patient satisfaction and emotional intelligence skills of nurses working in surgical clinics. Patient Preference and Adherence, 11, 1363-1368.

3. Champagne, F., Guisset, A.L., Veillard, J., \& Trabut, I. Performance Assessment Tool for Quality Improvement in Hospitals (PATH). (2005). Available: http://www.irspum.umontreal.ca/rapportpdf/R0506.pdf

4. Consumer Assessment of Healthcare Providers and Systems (CHAPS). Available: https://www.cms.gov/Research-Statistics-Data-and-Systems/Research/CAHPS/

5. Ferrand, Y., Siemens, J., Weathers, D., Fredendall, L.D., Choi, Y., Pirrallo, R.G., \& Bitner, M. (2016). Patient Satisfaction with Healthcare Services: A Critical Review. Quality Management Journal, 23(4), 6-22.

6. Fitzpatrick, R. (1997). The assessment of patient satisfaction. In C. Jenkinson Assessment and evaluation of health and medical care. Buckingham: Open University Press, 85-101.

7. Golder, p. N., Mitra, D., \& Moorman, C. 2012. What is quality? An integrative framework of processes and states. Journal of Marketing, 76, 1-12.

8. Kumar, P., Sasadhar Bera, S., \& Chakraborty, S. (2017). An Examination of the Association between Service Convenience Flexibility in Healthcare Delivery Systems and Patient Satisfaction. South Asian Journal of Management, 24(4), 35-54.

9. Linder-Pelz, S. (1982). Social psychological determinants of patient satisfaction: a five test hypothesis. Social Science and Medicine, 16, 583-589.

10. Okpala, p. (2018). Assessment of the influence of technology on the cost of healthcare service and patient's satisfaction. International Journal of Healthcare Management, 11(4), 351-355.

11. Parasuraman, A., Zeithaml, V. A.,\& Berry, L. L. (1985). A conceptual model of service quality and its implications for future research. Journal of Marketing, 49(4), 41-50.

12. Ponsignon, FreWilliams, M., \& Hall, J. (2015). Healthcare experience quality: an empirical exploration using content analysis techniques. Journal of Service Management, 25(3), 3-29.

13. Radu, G., Maria Radu, M., Andrei Condurache, A., \& Victor Lorin Purcarea, V.L. (2018). Managerial strategies and policies - Analysis of patient satisfaction based on demographic data. Journal of Medicine and Life, 11(3), 218-224.

14.Sergio, A. (2015). A model of antecedents for the co-creation of value in health care: An application of Structural Equation Modeling, Brazilian Business Review, 12(6), 121-149.

15.Sfantou, D.E., Laliotis, A., Patelarou, A.E., Sifaki-Pistolla, D., Matalliotakis, M., \& Patelarou, E. (2017). Importance of Leadership Style towards Quality of Care Measures in Healthcare Settings: A Systematic Review. Healthcare, 5(4), 73.

16. The consolidated version of the contract about Foundation of the European Union and European Community. (2007). Available: http://www.ecb.europa.eu/ecb/legal/pdf/ce32120061229lv00010331.pdf

17. The Constitution of the Republic of Latvia. (1922). Available: http://likumi.Iv/doc.php?id=57980 
18. Valencia-Arias, A., Bran-Piedahita, L., Botero-Zapata, Benjumea, M., \& Palacios-Moya, L. (2018). Mapping the Healthcare Service Quality Domain: A Bibliometric Analysis. Journal of Clinical and Diagnostic Research, 12(8), 1-5.

19. Wang, H., Kline, J.A., Jackson, B.E., Laureano-Philips, J., Robinson, R.D., Cowden, C.D., d'Etienne, J.P., Arze, S.E., \& Zenarosa, N.R. (2018). Association between emergency physician self-reported empathy and patient satisfaction. PLOS ONE, 13,1-12. 